\title{
Transverse sound in a magnetic field in $\mathrm{UPt}_{3}$
}

\author{
B. S. Shivaram, ${ }^{*}$ Y. H. Jeong, ${ }^{\dagger}$ and T. F. Rosenbaum \\ James Franck Institute and the Department of Physics, The University of Chicago, Chicago, Illinois 60637 \\ D. G. Hinks \\ Materials Science and Technology Division, Argonne National Laboratory, Argonne, Illinois 60439 \\ S. Schmitt-Rink \\ AT\&T Bell Laboratories, Murray Hill, New Jersey 07974 \\ (Received 11 December 1986)
}

\begin{abstract}
We have propagated transverse sound in a magnetic field in the basal plane of $\mathrm{UPt}_{3}$, with the polarization vector oriented both in the basal plane and perpendicular to it. We observe a strong anisotropy in the magnetic field dependence of the attenuation for the two polarizations. Using a simple phenomenological model, we can understand the low-temperature field dependence as a natural consequence of the anisotropy with temperature in zero field reported earlier [Phys. Rev. Lett. 56, 1078 (1986)]. However, for increasing temperatures there are significant deviations from this model. In no case do we find evidence for new superconducting phases in a magnetic field.
\end{abstract}

Propagation of sound in heavy-fermion superconductors has received much experimental attention recently. ${ }^{1-3}$ The salient features observed in these experiments are the various power-law dependences observed for the attenuation of both longitudinal and transverse sound waves and the peak in the attenuation observed near $T_{c}$ for longitudinal sound only. The power-law dependences in particular have been interpreted as proof for the presence of nodes in the gap. ${ }^{4-6}$ However, the observation of a mere power-law dependence in a physical quantity does not suffice to define the anisotropic state completely. It is more useful to seek a direct manifestation of gap anisotropy by measuring the same quantity in various directions. One such work was the measurement of transverse sound reported in Ref. 3. In this experiment an approximately linear temperature dependence of the attenuation was observed when both the wave vector and the polarization were in the basal plane. However, a rotation of the polarization $\varepsilon$ alone by $\pi / 2$ resulted in a superlinear temperature dependence of the attenuation. It was suggested by Pethick and Pines ${ }^{7}$ that the calculation of transport properties in heavy-fermion superconductors should be performed in the limit where phase shifts due to impurity scattering are close to $\pi / 2$. Using this suggestion, the attenuation of ultrasound was calculated in a self-consistent manner ${ }^{8,9}$ and the anisotropy of transverse sound was interpreted as evidence for a polarlike state with the lines of zeroes in the gap present in the basal plane. A comparison of axial-like and polarlike states has been made in Refs. 10 and 11 , with different conclusions drawn.

While it is likely that the controversy about the specific nature of the gap state will continue for some time, it is useful to further enumerate the anisotropic features of the superconducting state in $\mathrm{UPt}_{3}$. In this paper, we report on the anisotropy of the magnetic field dependence of transverse sound attenuation. We can relate the magnetic- field-dependent anisotropy to the temperature-dependent anisotropy previously reported ${ }^{3}$ for zero field. Furthermore, we explore the possibility that the magnetic field can influence the superconducting order parameter and induce new forms of superconductivity.

In our work the wave vector $\mathbf{q}$ was parallel to the $b$ axis and the polarization $\varepsilon$, was either along the $a$ or the $c$ axis of $\mathrm{UPt}_{3}$. The magnetic field $\mathbf{H}$ was held parallel to $\mathbf{q}$. We note that the only difference between the two cases measured is the orientation of the polarization with respect to the crystalline axes. The same physical sample was used in separate runs with only the orientation of the quartz transducer varied.

In Fig. 1 we show the increase in attenuation $\alpha$ as a function of magnetic field $H$ for the two polarizations studied at $T=100 \mathrm{mK}$. For the orientation with $\boldsymbol{\varepsilon} \| \mathbf{a}$, an increase which starts linearly develops a pronounced curvature for fields toward the upper critical field $H_{c 2}$. Motivated by an earlier theory ${ }^{12}$ of sound attenuation in type-II superconductors, we have been able to fit our results well to the form $\Delta \alpha(H) \sim\left[1-\left(1-H / H_{c 2}\right)^{1 / 2}\right]$. The values of $H_{c 2}$ deduced from such a fit are the same as those measured by us earlier. ${ }^{13}$ We find different behavior, however, in the orthogonal case. For $\varepsilon \| \mathbf{c}$, the increase in attenuation is consistent with a linear fit $\Delta \alpha(H) \sim[1-(1$ $\left.-H / H_{c 2}\right)$ ] all the way up to $H_{c 2}$.

In Fig. 2 we show the temperature dependence of the attenuation measured on the same sample for which the magnetic field dependence is shown in Fig. 1. Fitting the results all the way from 0 to $T_{c}$ we find the best fit exponents $1.1 \pm 0.1$ and $2.1 \pm 0.2$ for the cases $\boldsymbol{\varepsilon} \| \mathbf{a}$ and $\boldsymbol{\varepsilon} \| \mathbf{c}$, respectively (solid lines). However, a word of caution regarding the power laws used is appropriate here. Theory has shown that ${ }^{9-11}$ the attenuation in anisotropic superconductors is only approximated by power laws. In addition, the power laws are dependent on the temperature in- 


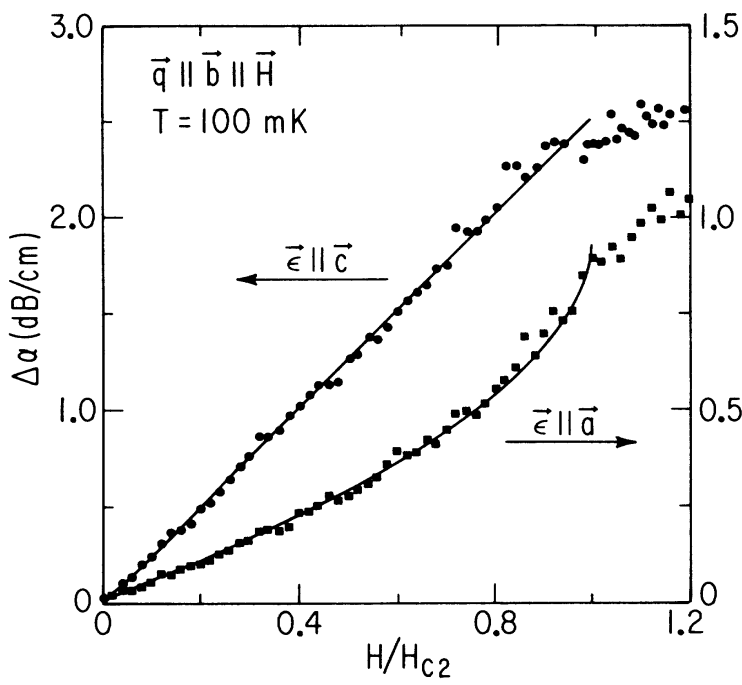

FIG. 1. Increase in the attenuation of transverse sound at 132 $\mathrm{MHz}$ with magnetic field for both polarizations $\boldsymbol{\varepsilon} \| \mathbf{c}$ and $\boldsymbol{\varepsilon} \| \mathbf{a}$. The line through the circles $(\varepsilon \| \mathrm{c})$ is a fit to $\Delta \alpha(H)=a[1-(1$ $\left.\left.-H / H_{c 2}\right)\right]$ and the line through the squares $(\varepsilon \| \mathbf{a})$ is a fit to $\alpha(H)=b\left[1-\left(1-H / H_{c 2}\right)^{1 / 2}\right]$ with $H_{c 2}=2.4 \mathrm{~T}$.

tervals used to fit the data and also the extent and nature of the impurities present. This is substantiated by the considerable variation in results obtained by different groups for the attenuation of longitudinal sound. 1,2

The dashed lines in Fig. 2 are the full functional forms for the temperature dependence of the attenuation from Schmitt-Rink, Miyake, and Varma, ${ }^{9}$ assuming a polarlike

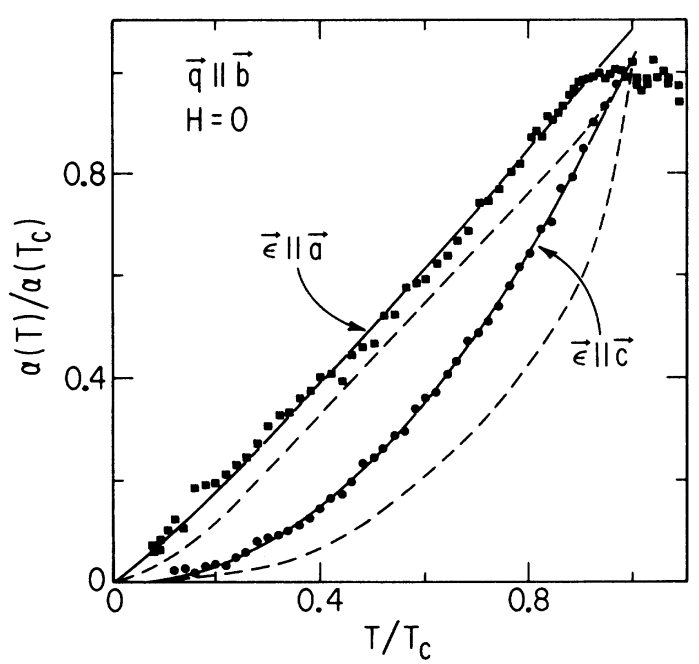

FIG. 2. The temperature dependence of the attenuation normalized to the value at $T_{c}$ for both polarizations ellc and ella measured on the same sample as in Fig. 1. The solid lines drawn through the squares $(\varepsilon \| \mathbf{a})$ and circles $(\varepsilon \| \mathbf{c})$ are power-law fits to the entire data in the superconducting state with best-fit exponents 1.1 and 2.1 , respectively. The dashed line is the full functional form for a polarlike state from Ref. 9. state with the line of nodes of the order parameter present in the basal plane. The theory is for impurity scattering in the unitarity limit, the normal-state impurity scattering rate $\tau_{n}^{-1}$ being $0.02 \tau_{c}^{-1}$, where $\tau_{c}^{-1}$ is the critical rate for the vanishing of superconductivity. The agreement with the experimental data can be improved if one considers the possibility that a small part of the sample stays normal. ${ }^{9}$ This introduces a small constant background so that the theoretical curves are shifted up.

In analyzing critical field measurements in type-II superconductors, it has been conventional for a long time to plot the deviation of the observed results from the quadratic dependence expected in a simple two-fluid model. ${ }^{14,15}$ Thus we may define the parameter $\delta$,

$$
\delta=\left[\frac{H_{c 2}}{H_{c 2}(0)}-\left(1-\frac{T^{2}}{T_{c 0}^{2}}\right)\right] .
$$

In the inset of Fig. 3 we show the quantity $\delta$ obtained from our critical-field measurements in the basal plane. ${ }^{13} \mathrm{We}$ note that at the lowest temperatures, $T \leq 100 \mathrm{mK}, \delta \approx 0$. Using the measured temperature dependence in zero field

$$
\begin{aligned}
\alpha / \alpha_{N} & =T / T_{c 0}, \boldsymbol{\varepsilon} \| \mathbf{a} \\
& \simeq\left(T / T_{c 0}\right)^{2}, \boldsymbol{\varepsilon} \| \mathbf{c},
\end{aligned}
$$

and with the reasonable assumption that the pair-breaking magnetic field changes predominantly the zero-termperature values of $\alpha$ but leaves its slope with temperature unchanged, we obtain from Eqs. (1) and (2) for $H<H_{c 2}$,

$$
\begin{aligned}
\Delta \alpha / \alpha_{N} & =1-\left[1-H / H_{c 2}(0)\right]^{1 / 2}, \boldsymbol{\varepsilon} \| \mathbf{a} \\
& \simeq 1-\left[1-H / H_{c 2}(0)\right], \quad \boldsymbol{\varepsilon} \| \mathbf{c},
\end{aligned}
$$

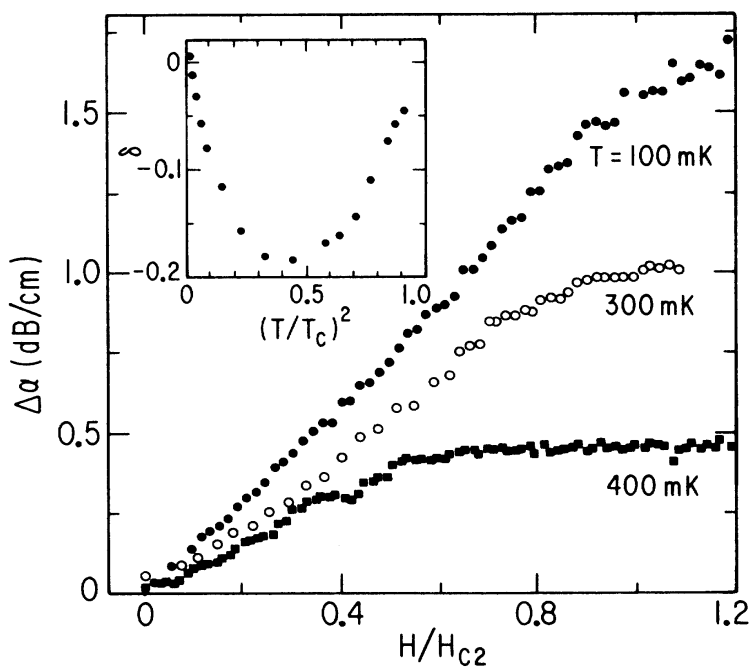

FIG. 3. Increase in attenuation with magnetic field for $\varepsilon \| c$ at three different temperatures. The inset shows the deviation $\delta$ of the upper critical field from the behavior defined by Eq. (1). Note that for the lowest temperatures $T \leq 100 \mathrm{mK}, \delta \simeq 0$, and the increase in attenuation is nearly linear for $H$ up to $H_{c 2}$. But at higher $T$ and larger values of $\delta$, a knee develops in the magnetic field dependence of the attenuation. 
where $\alpha_{N}$ is the normal-state value of $\alpha$. At low temperatures, where $H_{c 2}=H_{c 2}(0)$, these expressions indeed describe the observed behavior without any adjustable parameter.

Our results for higher $T$ are not so easy to understand. The main part of Fig. 3 shows the increase in attenuation, for the geometry with $\boldsymbol{\varepsilon} \| \mathbf{c}$ at three different temperatures. The overall behavior is still described by Eq. (3), but at $T=300 \mathrm{mK}$ the change in attenuation flattens already for $H>0.8 H_{c 2}$ and at $T=400 \mathrm{mK}$ it flattens for even lower fields. This might be explained by the rounding of the superconducting transition and the more complicated behavior of $H_{c 2}$. As shown in the inset of Fig. 3, the measured critical fields deviate from the parabolic law [Eq. (1)] at temperatures greater than $T=100 \mathrm{mK}$. For these temperatures, the sample achieves its normal state for fields lower than those given by Eq. (1), which necessitates rescaling the magnetic field. We await detailed calculations of sound attenuation in anisotropic superconductors in a magnetic field for a more proper analysis.

Measurements of the ultrasound attenuation in a magnetic field have also been performed by Müller et al. ${ }^{16} \mathrm{~A}$ peak in the attenuation of longitudinal sound observed significantly below $H_{c 2}$ and hysteresis effects observed in low fields in the superconducting state has been attributed to the presence of two stable and one metastable phase induced by a magnetic field. Such a variety of superconducting phases can arise only in the event of the angular momentum $l>0$ for the Cooper pairs. ${ }^{17}$ If true, similar structure should be visible in the attenuation of transverse sound. In the present work neither a peak below $H_{c 2}$ nor any hysteresis effects have been observed. We also find monotonic, reversible behavior for $\mathbf{H} \| \mathbf{c}$. We note that hysteresis in measuring attenuation in low fields has been seen even in ordinary superconductors and the effect has been attributed to flux pinning. ${ }^{18}$ It thus appears to us that claims for the observation of more than one superconducting phase in $\mathrm{UPt}_{3}$ are presently unwarranted.

In summary, we have observed a unique anisotropy in the magnetic field dependence of transverse sound attenuation in $\mathrm{UPt}_{3}$, which depends purely on the orientation of the polarization with respect to the crystalline axes. We have shown that these observations are a consequence of our earlier work which revealed an anisotropy of the temperature dependence of the attenuation in zero field. The application of a magnetic field either in or perpendicular to the basal plane does not appear to induce new forms of superconducting order.

We should like to acknowledge helpful discussions with S. R. Nagel, K. Quader, C. M. Varma, and P. Wölfle. One of us (B.S.S.) thanks K. Scharnberg for useful correspondence. The work at Argonne National Laboratory was supported by the U. S. Department of Energy under Contract No. W-31-109-ENG-38. The work at The University of Chicago was supported by the National Science Foundation under Grant No. DMR 8351992. One of us (T.F.R.) acknowledges partial financial support from the Alfred P. Sloan Foundation.

*Present address: Department of Physics, University of Virginia, Charlottesville, VA 22901.

†Present address: Department of Chemistry, Massachusetts Institute of Technology, Cambridge, MA 02139.

${ }^{1}$ D. J. Bishop, C. M. Varma, B. Batlogg, E. Bucher, Z. Fisk, and J. L. Smith, Phys. Rev. Lett. 53, 1009 (1984).

${ }^{2}$ V. Müller, D. Maurer, E. W. Scheidt, C. Roth, K. Luders, E. Bucher, and H. E. Bommel, Solid State Commun. 57, 319 (1986).

${ }^{3}$ B. S. Shivaram, Y. H. Jeong, T. F. Rosenbaum, and D. G. Hinks, Phys. Rev. Lett. 56, 1078 (1986).

${ }^{4}$ G. R. Stewart, Rev. Mod. Phys. 56, 755 (1984).

${ }^{5}$ C. M. Varma, Comments Condens. Matter Phys. 11, 221 (1985).

${ }^{6}$ Z. Fisk, H. R. Ott, T. M. Rice, and J. L. Smith, Nature (London) 320, 124 (1986).

${ }^{7}$ C. J. Pethick and D. Pines, Phys. Rev. Lett. 57, 118 (1986).

${ }^{8}$ P. Hirschfeld, D. Vollhardt, and P. Wolfle, Solid State Commun. 59, 111 (1986).

${ }^{9}$ S. Schmitt-Rink, K. Miyake, and C. M. Varma, Phys. Rev. Lett. 57, 2575 (1986).

${ }^{10}$ H. Monien, K. Scharnberg, L. Tewordt, and D. Walker (unpublished).

${ }^{11}$ K. Scharnberg, D. Walker, H. Monien, L. Tewordt, and R. A. Klemm (unpublished).

${ }^{12}$ K. Maki, Phys. Rev. 156, 437 (1967).

${ }^{13}$ B. S. Shivaram, T. F. Rosenbaum, and D. G. Hinks, Phys. Rev. Lett. 57, 1259 (1986).

${ }^{14}$ J. R. Schrieffer, Theory of Superconductivity (Benjamin, New York, 1964), p. 5.

${ }^{15}$ B. S. Chandrasekhar, in Superconductivity, edited by R. D. Parks (Dekker, New York, 1969), p. 1.

${ }^{16}$ V. Müller, Ch. Roth, D. Maurer, E. W. Scheidt, and K. Luders (unpublished).

${ }^{17}$ A. J. Leggett, Rev. Mod. Phys. 47, 331 (1975).

${ }^{18}$ M. Gottlieb, M. Garbuny, and C. K. Jones, in Physical Acous tics, edited by W. P. Mason and R. N. Thurston (Academic, New York, 1970), Vol. III, p. 2. 